\title{
Sonografisch assistierte venöse Thrombektomie im ersten Trimenon - ein Fallbericht
}

\section{Sonographically assisted venous thrombectomy in the first trimester - A case report}

\author{
Autoren \\ Thomas Hummel ${ }^{1,2}$, Barbara Strohmann 1, 2, Thomas Falkenstein ${ }^{1,2}$, Markus Stücker², Achim Mumme $^{1,2}$, \\ Dominic Mühlberger ${ }^{1,2}$
}

Institute

1 Klinik für Gefäßchirurgie, Katholisches Klinikum Bochum der Ruhr-Universität Bochum

2 Venenzentrum der Dermatologischen und Gefäßchirurgischen Kliniken des Katholischen Klinikums Bochum der Ruhr-Universität Bochum

Schlüsselwörter

operative venöse Thrombektomie, Schwangerschaft, erstes Trimester, Ultraschall

Key words

operative venous thrombectomy, pregnancy, first trimester, ultrasound

online publiziert 29.04 .2021

Bibliografie

Phlebologie 2021; 50: 202-207

DOI 10.1055/a-1390-1168

ISSN 0939-978X

(C) 2021. Thieme. All rights reserved.

Georg Thieme Verlag KG, Rüdigerstraße 14,

70469 Stuttgart, Germany

Korrespondenzadresse

Dr. med. Thomas Hummel

Klinik für Gefäßchirurgie

St.-Josef-Hospital Bochum

Klinikum der Ruhr-Universität-Bochum, Gudrunstraße 56,

44791 Bochum, Deutschland

Tel.: +49/2 34/50922 70

Thomas.Hummel@ruhr-uni-bochum.de

\section{ZUSAMMENFASSUNG}

Es wird der Fall einer Patientin in der 12. Schwangerschaftswoche (SSW) mit einer deszendierenden 4-Etagen-Thrombose, welche unter konservativen stationären Maßnahmen einen Progress des Befundes erlitten hat, vorgestellt und diskutiert. Die Patientin und ihr Ehemann hatten sich trotz erhöhten Risikos für den Fötus für eine operative Rekanalisation entschieden. Die venöse Thrombektomie wurde sonografisch assistiert, ohne Anlage einer AV-Fistel, durchgeführt. Die bei Schwangeren eingeschränkten diagnostischen Möglichkeiten und Einflussfaktoren für eine Risikoevaluation für Mutter und Kind werden dargestellt.

\section{ABSTRACT}

The case of a patient in the $12^{\text {th }}$ week of pregnancy with a descending pelvic thrombosis, who suffered a progression of the thrombosis in spite of conservative, inpatient stay therapy, is presented and discussed. Despite the increased risk, the patient opted for surgical recanalization. The venous thrombectomy was successfully performed with sonography assistance. The diagnostic possibilities and influencing factors for a risk evaluation for mother and child, which are limited in pregnant women, are presented.

\section{Anamnese und Befunde}

Wir berichten über eine 33-jährige Patientin in der 12. Schwangerschaftswoche, die uns aus einer auswärtigen Gynäkologie vorgestellt wurde. Die Patientin befand sich bereits seit einer Woche in stationärer Behandlung mit einer Beckenvenenthrombose unter konservativen Therapiemaßnahmen. Unter einer therapeutischen Antikoagulation mit niedermolekularem Heparin und konsequenter Kompressionstherapie kam es allerdings zu einer Verschlechterung der Symptomatik mit ausgeprägter Schwellung des linken Beins, sodass die Patientin in unserer Klinik zur Beurteilung vorgestellt wurde.

Wir sahen eine schlanke Patientin in gutem Allgemeinzustand, ohne relevante Vorerkrankungen. Luftnot oder Hustenreiz wurden von der Patientin verneint. Diagnostisch bestand nach sonografischen Kriterien eine deszendierende 4-Etagen-Throm- 


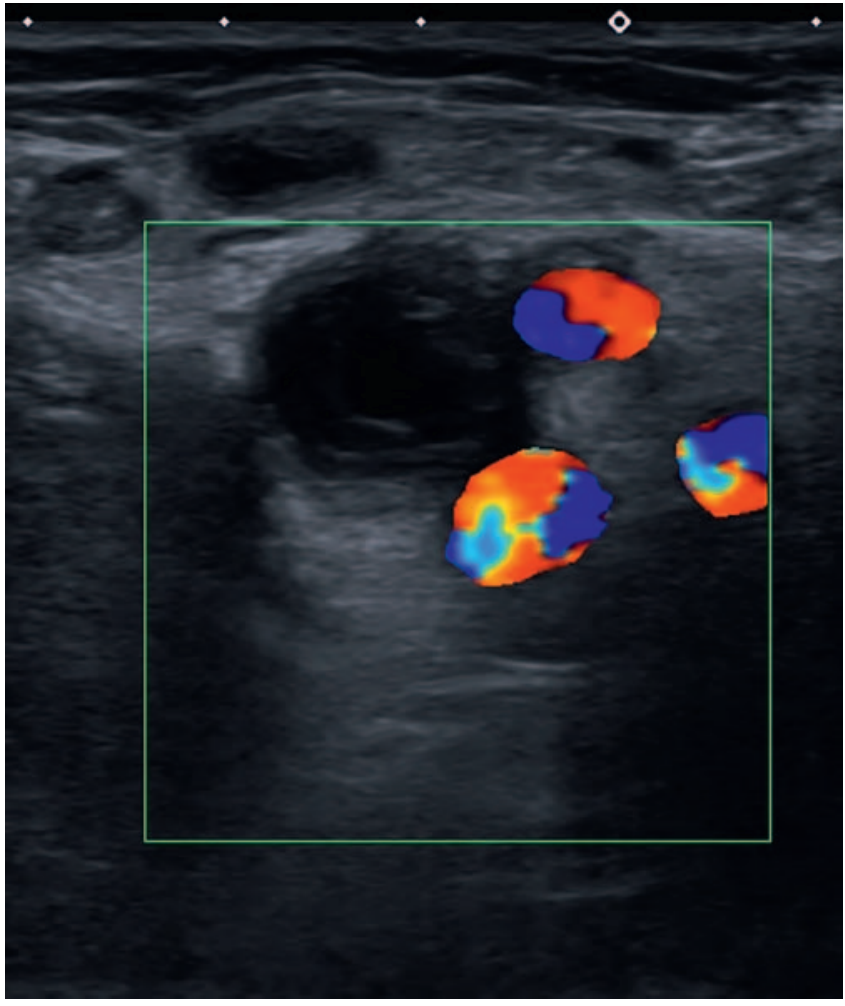

- Abb. 1 Darstellung der thrombosierten V. femoralis communis links.

bose. Passend hierzu zeigte sich ein inhomogener Thrombus der gesamten linken Beckenetage, welcher zum Leistenband zunehmend echoarm und dann nach distal bis in die V. poplitea reichend komplett echoarm zur Darstellung kam. A Abb. 1 zeigt den Thrombus im Bereich der V. femoralis communis des linken Beins.

Die V. cava inferior war frei darstellbar bei ansonsten komplett verschlossener Iliakal-, Femoral- und Poplitealstrombahn. Aufgrund des schlanken Habitus der Patientin war insgesamt eine sehr gute Beurteilbarkeit der venösen Strombahn, auch der zentralen Anteile, im Ultraschall gegeben. Klinische Hinweise für eine Lungenembolie bestanden nicht. Des Weiteren bestand kein Hinweis für eine Phlegmasia coerulea dolens bei gut tastbaren Fußpulsen und triphasischen Dopplersignalen.

\section{Risikoevaluation für Diagnostik und Therapie}

Grundsätzlich ist bei Thrombosen während einer Schwangerschaft immer zwischen dem mütterlichen und dem kindlichen Risiko zu differenzieren. Das durch eine Narkose bestehende Risiko scheint nicht mit einem schlechteren Outcome für Mutter und Kind verbunden zu sein und ist v. a. durch Erfahrungen im Rahmen von Appendektomien empirisch analysiert worden [1-3]. Neben den allgemeinen Risiken der Operation wie Wundheilungsstörung, Infekt, Blutung oder Verletzung von Begleitstrukturen liegt das Hauptrisiko in der Diagnostik und Therapie bei schwangerschaftsassoziierten Thrombosen in der maternen und fetalen Belastung durch die Röntgenstrahlung. Einen wichtigen Schwellenwert stellt eine Strahlenbelastung von $50 \mathrm{mSv}$ dar. So sind in empirischen Untersuchungen bei einer Strahlenbelastung von weniger als 50 mSv keine strahlenassoziierten aberrierenden Schwangerschaftsverläufe nachweisbar, während eine Strahlenbelastung von 50-100 mSv innerhalb der ersten 2 Wochen der Schwangerschaft häufig zum Abort führt. Der Fötus ist während der Organogenese in der 8.-10. Schwangerschaftswoche besonders strahlensensibel; so führen Strahlenbelastungen über 100-200 mSv häufig zu genetischen Malformationen, intrauterinen Wachstumsstörungen, Intelligenzminderungen sowie Aborten. Zusätzlich führt die Strahlenbelastung zu einer stochastischen Wahrscheinlichkeit einer Karzinomentwicklung im Laufe des weiteren Lebens [4-8].

Aufgrund dessen ist die Diagnostik einer venösen Thromboembolie bei Schwangeren stark eingeschränkt - so ist ein venöses Angio-CT mit einer hohen Strahlenbelastung von 10-50 mSv für Mutter und Kind vergesellschaftet [8]. Das stochastische Risiko von Kindheitskarzinomen bis zum 15. Lebensjahr ist erhöht. Wenige Daten sind zur MRT-Sicherheit in der Organogenese bekannt. Gadolinium passiert die Plazentaschranke, wird vom Fötus aufgenommen und dessen Halbwertzeit im Fötus ist unklar. Allerdings zeigt Gadolinium in Tierversuchen einen potenziell teratogenen Effekt [8, 9]. Daher ist eine MR-Diagnostik grundsätzlich bei Thrombosen in der Schwangerschaft restriktiv empfohlen, eine Gadolinium-Gabe sollte vermieden werden. Untersuchungen mittels kontrastloser Time-of-flight-MRA-Untersuchungen sind in der Indikation bei Schwangeren bisher nicht verfügbar.

In der Diagnostik einer Lungenembolie mittels PulmonalisAngio-CT besteht ein Ungleichgewicht in der Strahlendosis, da der Uterus nicht im Strahlengang der Untersuchung liegt. So hat die Mutter eine Strahlenbelastung von 7-70 mSv und das Kind von 0,01-0,66 mSv. Die Mutter hat bei der Untersuchung während der Schwangerschaft und Stillzeit ein besonders hohes Risiko für die stochastische Entwicklung eines Mammakarzinoms aufgrund der proliferierenden Brustdrüse [5]. Anteile des Jod-haltigen Kontrastmittels sind zudem plazentagängig und belasten den kindlichen Schilddrüsenstoffwechsel.

In den therapeutischen Optionen neben der rein konservativen Therapie ist der Behandler ebenfalls limitiert. So scheiden herkömmliche endovaskuläre Verfahren aufgrund der notwendigen Röntgendurchleuchtung zur Katheter-Positionierung und Lyseverfahren aufgrund der bestehenden Schwangerschaft weitestgehend aus. Auch die operative Therapie ist mit unterschiedlichen Strahlendosen in herkömmlicher Technik belastet.

\section{Verlauf}

Entsprechend einer maternen und fetalen Risiko-Nutzen-Evaluation sahen wir am Ende des 1. Trimenons das Hauptrisiko für eine Thrombus-beseitigende Therapie auf der Seite des Kindes, insbesondere durch ein hohes Risiko für eine Strahlenexposition aufgrund der fraglich noch nicht abgeschlossenen Organogenese. Wir sahen ein geringes Risiko durch eine Narkose. Auch das durch die Anamnese anzunehmende Alter der Thrombose von mindestens 1 Woche wurde als kritisch bewertet. Entsprechend wurden die Eltern aufgeklärt und eine Empfehlung zur Fortsetzung der konservativen Behandlung gegeben. Aufgrund der ausgeprägten 
Klinik, welche unter der konservativen Therapie progredient war, entschlossen sich die Eltern allerdings für die Durchführung einer rekanalisierenden Therapie.

Es erfolgte eine transfemorale operative Thrombektomie unter störungsfreier Narkose. Aufgrund der hervorragenden Ultraschallbedingungen bei der schlanken Patientin der zentralen-venösen Strombahn erfolgte die venöse Thrombektomie sonografisch assistiert. Der Fogarty-Katheter ließ sich sehr gut in der V. cava inferior und den Beckenvenen visualisieren. Die $>$ Abb. 2, 3 zeigen den venösen Thrombektomie-Katheter in der Cava und linken Vena iliaca communis.

Allerdings bestand der hochgradige Verdacht auf einen beim Rückzug des Katheters ertastbaren Residualthrombus im Bereich der V iliaca communis, passend zur Verdachtsdiagnose einer deszendierenden Thrombose. Es erfolgte zunächst der Einsatz eines Ringstrippers, ebenfalls sonografisch assistiert. $>$ Abb. 4 zeigt das Ultraschallbild des Ringstrippers im Bereich der V. iliaca communis.

Der Verdacht auf eine Unregelmäßigkeit im Bereich der Communis bestand allerdings abgeschwächt weiterhin, sodass wir uns bei vermutetem Residualthrombus zur phlebografischen Kontrolle entschlossen. Es erfolgte sonografisch assistiert zur besseren Visualisierung der Vene die Einlage eines Führungsdrahtes. A Abb. 5 zeigt die gute Sichtbarkeit des Terumo-Drahtes im Ultraschall.

Zur Uterus-Dosisreduktion erfolgte eine Positionierung des Durchleuchtungsgeräts mittels Positionslaser und Single-shotPositionierung und maximaler Einblendung. Auf die Durchführung eines DSA-Modus wurde verzichtet und die Pulsfrequenz maximal gedrosselt (2 Bilder/s). > Abb. 6 zeigt die so zur Darstellung kommende Phlebografie mit dem Verdacht auf einen wandadhärenten Residualthrombus der V. iliaca communis.

Die intraoperative Durchleuchtungszeit betrug insgesamt 3 Sekunden. Es erfolgte der erneute sonografisch assistierte Ringstripper-Einsatz mit Thrombektomie-Manöver, bei dem ein alter Thrombus aus der V. iliaca communis geborgen werden konnte. Hiernach bestand ein freier Abstrom, ohne ertastbares Hindernis oder Unregelmäßigkeit beim Rückzug des Katheters. Auf eine phlebografische Abschlusskontrolle wurde verzichtet. Anschließend erfolgte eine primär erfolgreiche Kompressions-Thrombektomie der Beinstrombahn mit Bergung eines langstreckigen Thrombus aus der Profunda und Femoro-/Poplitealvene. Auf die Anlage einer AV-Fistel wurde bei sehr gutem Einstrom aus den Beinvenen verzichtet.

Der weitere postoperative Verlauf war unter Frühmobilisation, medikamentöser Therapie mit niedermolekularem Heparin und Kompression komplikationslos. Die $>$ Abb. 7, 8 zeigen die postoperativen Kontrollen der Becken- und Oberschenkelstrombahn mit freiem tiefem Venensystem.

Die gynäkologischen Kontrollen waren unauffällig und der weitere Schwangerschaftsverlauf mit Geburt normal. > Abb. 9 zeigt die postoperative Sonografie des Fötus. Ein Jahr nach der Geburt zeigt das Kind eine normale Entwicklung ohne körperliche oder geistige Retardierung.

\section{Diskussion}

Mittlerweile ist, trotz der im Verlauf gesunkenen jährlichen Müttersterblichkeitsrate, die Thromboembolie eine der häufigsten

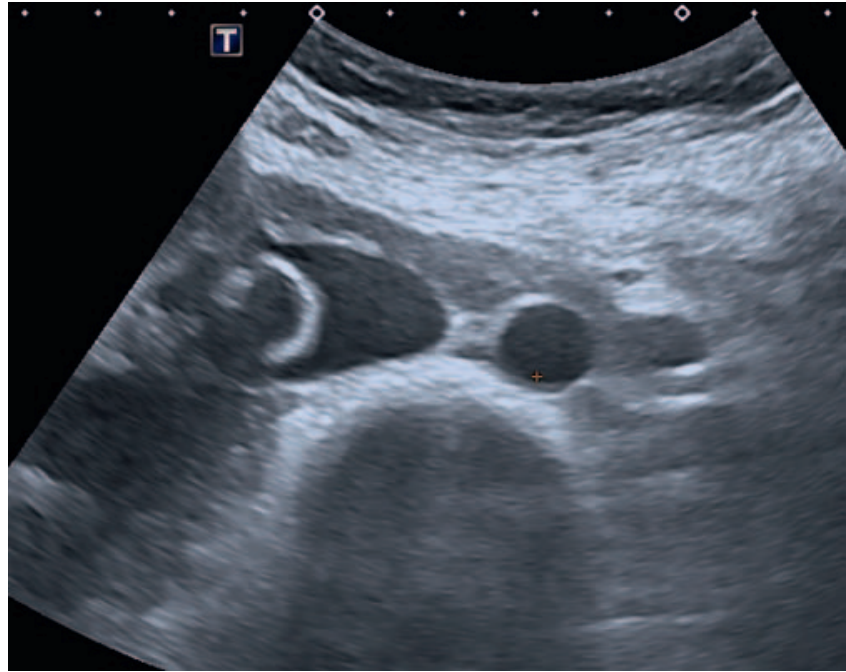

Abb. 2 Sonografie des Thrombektomie-Katheters in der V. cava inverior.

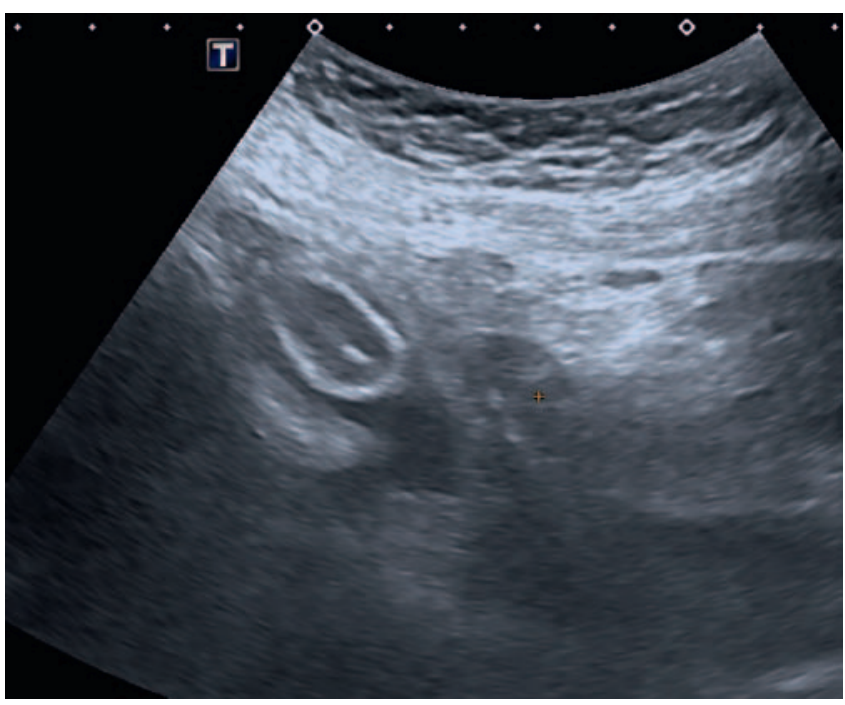

Abb. 3 Sonografie des Thrombektomie-Katheters in der V. iliaca communis.

schwangerschaftsassoziierten Todesursachen in den westlichen Industrieländern [10]. Die Inzidenz einer TVT während einer Schwangerschaft liegt bei 1:500-1500. Insgesamt besteht ein 5-fach erhöhtes Risiko einer TVT in der Schwangerschaft. Der Zeitpunkt der Diagnose einer TVT im Schwangerschaftsverlauf liegt hauptsächlich im dritten Trimenon. Vor allem in den letzten 2 Wochen vor der Geburt und im Wochenbett besteht ein deutlich erhöhtes Thromboserisiko [8, 11, 12].

Die Datenlage zu den rekanalisierenden Verfahren einer tiefen Becken-Bein-Venenthrombose bei Schwangeren ist stark limitiert und hat sich in der letzten Zeit nicht wesentlich verändert. Aufgrund dessen sind bei Schwangeren Thrombus-entfernende Verfahren nach den Empfehlungen der Society of Thrombosis and Haemostasis vor allem Extremitäten-bedrohenden Thrombosen vorbehalten [13]. Diese Empfehlung stützt sich allerdings haupt- 


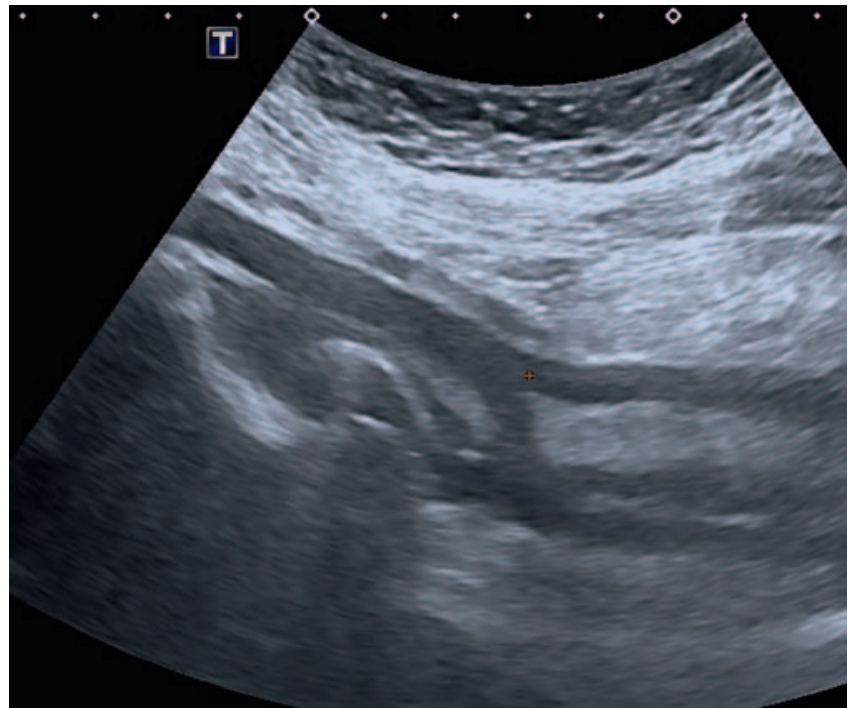

- Abb. 4 Sonografie des Ringstrippers in der V. iliaca communis.

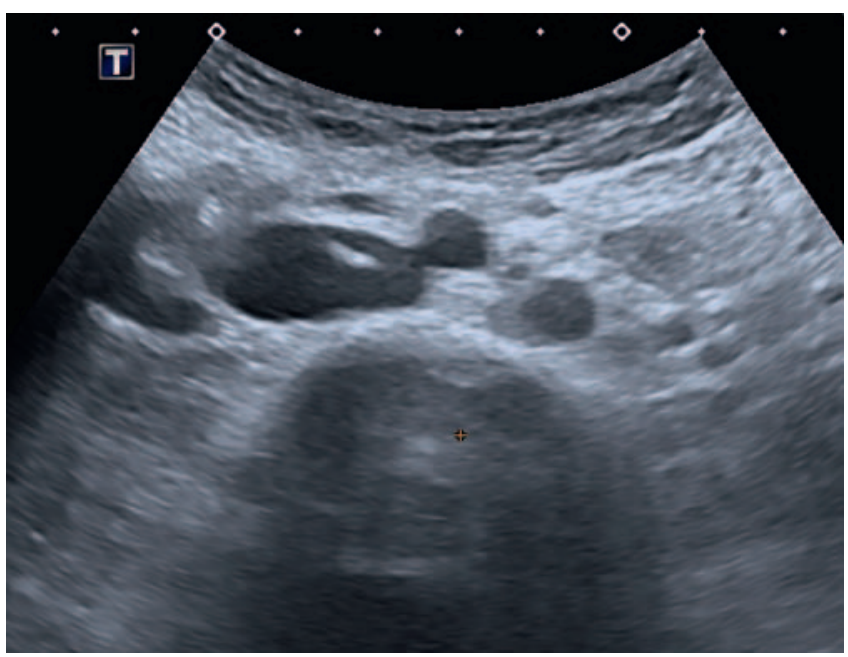

- Abb.5 Darstellung des Führungsdrahtes im Ultraschall.

sächlich auf Ergebnisse der interventionellen, Katheter-gestützten CaVenT-Studie, welche an Nichtschwangeren durchgeführt wurde [14], und einer fallzahlschwachen Untersuchung, bei der ebenfalls 11 der 13 Patienten mittels Katheter-gestützten Verfahren behandelt wurden [15]. Allerdings erscheint eine kumulative materne und fetale Komplikationsrate von $20 \%$ der Kathetergestützten Verfahren bei Schwangeren aus einem Review zusammengetragener Fallberichte aus dem Jahr 2018 relevant [16]. Eine Evaluation der bestehenden besseren Datenlage zur operativen Thrombektomie bei Schwangeren fand keinen Einzug in die Empfehlungen der Fachgesellschaft.

In unserem Fall bestand eine seltene Befundkonstellation mit einer deszendierenden TVT der Becken-Bein-Strombahn im ersten Trimenon mit deutlichem klinischem Progress unter konsequenten konservativen stationär überwachten Maßnahmen. Die Patientin und ihr Ehemann waren sich bereits vor unserer Konsultation offensichtlich einig, wie sich im Beratungsgespräch zeigte,

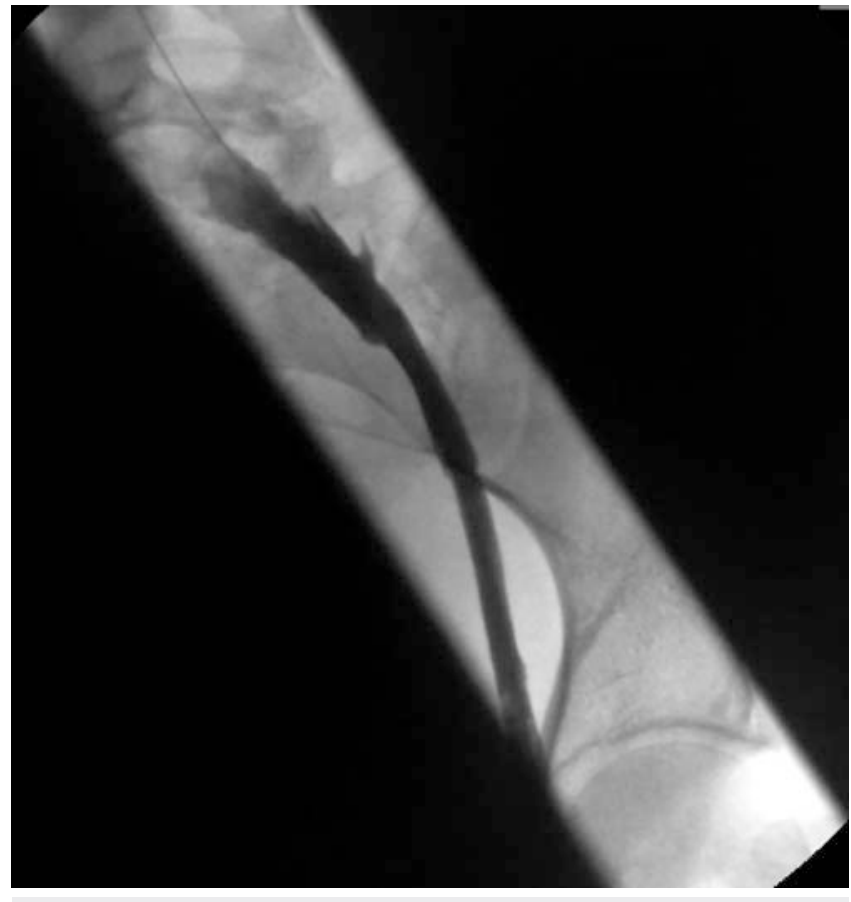

- Abb. 6 Intraoperative Phlebografie der Beckenvenen.

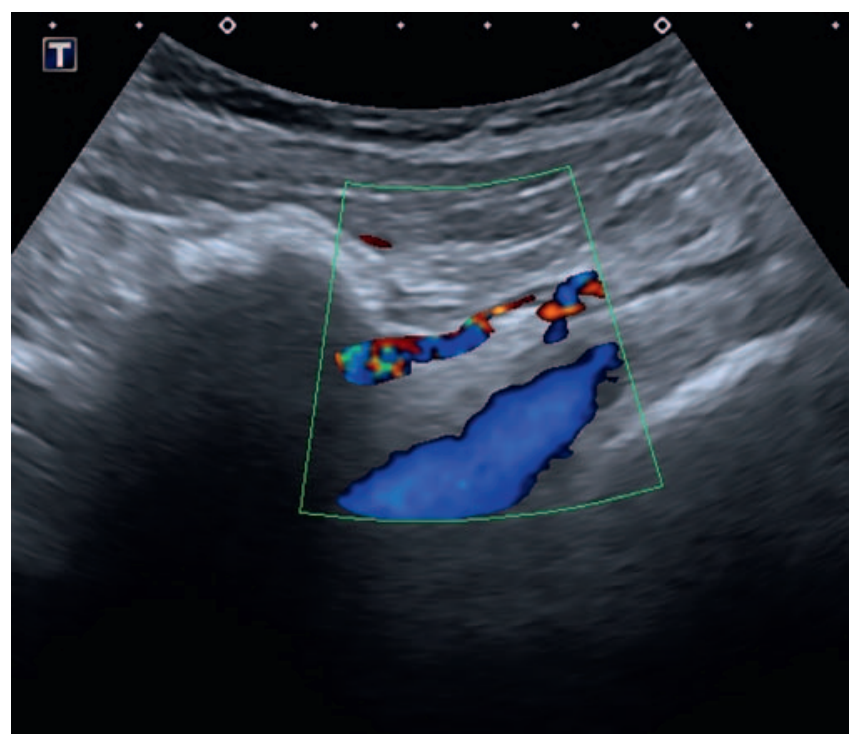

- Abb.7 Postoperative Duplexsonografie der Beckenvenen.

dass ein weiteres konservatives Vorgehen keine Option für sie darstellte, sodass wir wie ausgeführt aufgrund der hervorragenden Schallbedingungen ein sonografisch assistiertes operatives Vorgehen durchführten. In der beschriebenen Technik gelang es nahezu röntgenstrahlenfrei eine operative venöse Thrombektomie erfolgreich durchzuführen. Die technische Durchführung in der dargestellten Form ist im Wesentlichen abhängig von der sonografischen Assistenz/Expertise und erfordert unserer Ansicht nach ein dementsprechendes Setting. In der Rückschau würden wir heute auf die phlebografische Darstellung der Beckenvenen verzichten und stattdessen einen intravaskulären Ultraschall 


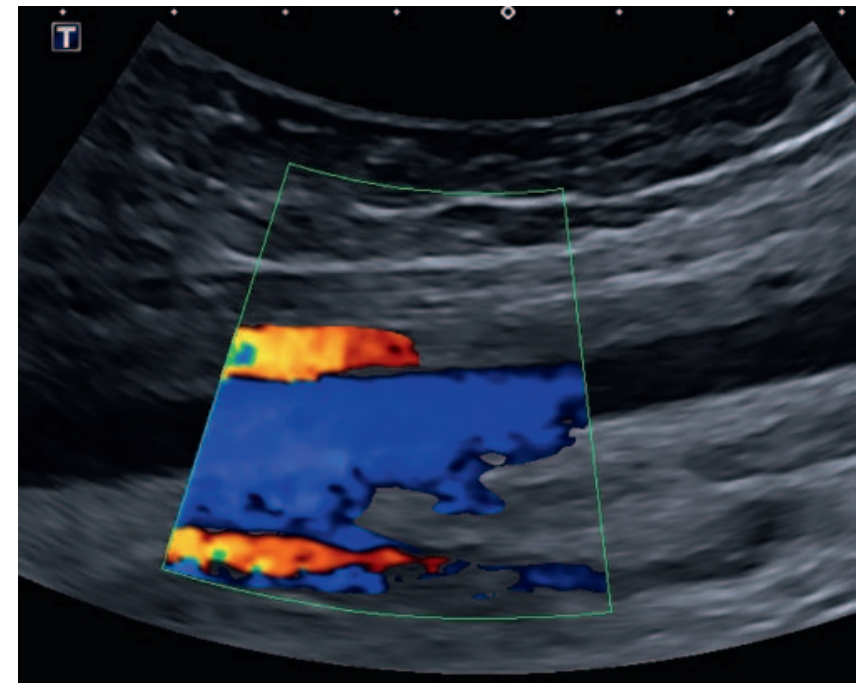

- Abb. 8 Postoperative Duplexsonografie der Femoralvene.

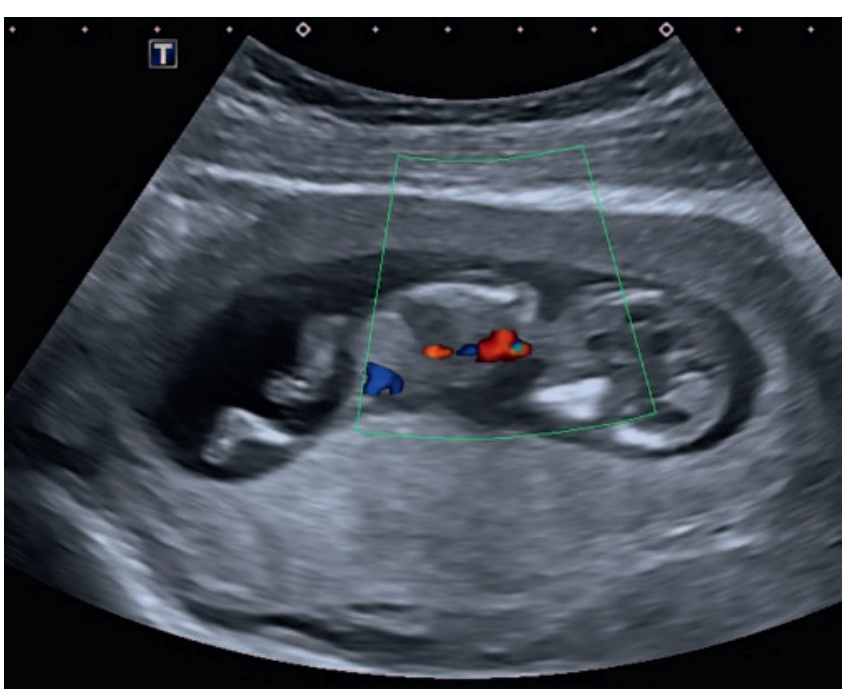

Abb. 9 Postoperative Sonografie des Fötus.

(IVUS) durchführen, um so eine visuelle Darstellung residueller Thromben oder Stenosen komplett ohne Röntgendiagnostik durchführen zu können.

Bisher ist in der Literatur keine sonografisch assistierte operative Thrombektomie im ersten Trimenon der Schwangerschaft beschrieben worden, sodass sich keine Rückschlüsse auf Indikation oder Vorgehen aus der Literatur herleiten lassen. Allerdings kann unserer Ansicht nach die dargestellte Technik in Abhängigkeit von den sonografischen Sichtverhältnissen zu einer wesentlichen Reduktion der Strahlenbelastung der Mutter und des Kindes beitragen.

Interessenkonflikt

Die Autorinnen/Autoren geben an, dass kein Interessenkonflikt besteht.
Danksagung

Dieses Manuskript wurde im Rahmen eines Habilitationsstipendiums der Ruhr-Universität Bochum (gefördert mit Mitteln der Firma Bauerfeind AG) erstellt.

Literatur

[1] Visser BC, Glasgow RE, Mulvihill KK et al. Safety and timing of nonobstetric abdominal surgery in pregnancy. Dig Surg 2001; 18 (5): 409-417. doi:10.1159/000050183. PMID: 11721118

[2] Vujic J, Marsoner K, Lipp-Pump AH et al. Non-obstetric surgery during pregnancy - an eleven-year retrospective analysis. BMC Pregnancy Childbirth 2019; 19 (1): 382 doi:10.1186/s12884-019-2554-6. PMID: 31653246; PMCID: PMC6815034

[3] Kuczkowski KM. The safety of anaesthetics in pregnant women. Expert Opin Drug Saf 2006; 5 (2): 251-264. PMID: 16503746 doi:10.1517| 14740338.5.2.251

[4] Niemann T, Nicolas G, Roser HW et al. Imaging for suspected pulmonary embolism in pregnancy-what about the fetal dose? A comprehensive review of the literature. Insights Imaging 2010; 1 (5): 361-372. doi:10.1007/s13244-010-0043-6. Epub 2010 Oct 2. PMID: 22347929; PMCID: PMC3259315

[5] International Commission on Radiological Protection. Pregnancy and medical radiation. Ann ICRP 2000; 30 (1): iii-viii, 1-43 doi:10.1016/ s0146-6453(00)00037-3. PMID: 11108925

[6] Fiebich M, Block A, Borowski M et al. Prenatal radiation exposure in diagnostic and interventional radiology. Rofo 2020. doi:10.1055/ a-1313-7527. Epub ahead of print. PMID: 33327031

[7] Tremblay E, Thérasse E, Thomassin-Naggara I et al. Quality initiatives: guidelines for use of medical imaging during pregnancy and lactation. Radiographics 2012; 32 (3): 897-911. Epub 2012 Mar 8. PMID: 22403117 doi: $10.1148 /$ rg.323115120

[8] Linnemann B, Bauersachs R, Rott $\mathrm{H}$ et al. Diagnosis of pregnancy-associated venous thromboembolism - position paper of the Working Group in Women's Health of the Society of Thrombosis and Haemostasis (GTH). Vasa 2016; 45 (2): 87-101. doi:10.1024/0301-1526/a000503. PMID: 27058795

[9] Puac P, Rodríguez A, Vallejo C et al. Safety of Contrast Material Use During Pregnancy and Lactation. Magn Reson Imaging Clin N Am 2017; 25 (4): 787-797. doi:10.1016/j.mric.2017.06.010. Epub 2017 Sep 8. PMID: 28964468

[10] Welsch H. Müttersterblichkeit während Geburt und Wochenbett bei vaginaler Entbindung und Sectio caesarea. Gynäkologe 1997; 30: 742756. doi:10.1007/PL00003001

[11] Heit JA, Kobbervig CE, James AH et al. Trends in the incidence of venous thromboembolism during pregnancy or postpartum: a 30-year population-based study. Ann Intern Med 2005; 143 (10): 697-706. doi:10.7326/0003-4819-143-10-200511150-00006. PMID: 16287790

[12] Pomp ER, Lenselink AM, Rosendaal FR et al. Pregnancy, the postpartum period and prothrombotic defects: risk of venous thrombosis in the MEGA study. J Thromb Haemost 2008; 6 (4): 632-637. doi:10.1111/ j.1538-7836.2008.02921.x. Epub 2008 Jan 31. PMID: 18248600

[13] Linnemann B, Scholz U, Rott $\mathrm{H}$ et al. Treatment of pregnancy-associated venous thromboembolism - position paper from the Working Group in Women's Health of the Society of Thrombosis and Haemostasis (GTH). Vasa 2016; 45 (2): 103-118. doi:10.1024/0301-1526/a000504. PMID: 27058796

[14] Enden T, Haig Y, Kløw NE et al. Long-term outcome after additional catheter-directed thrombolysis versus standard treatment for acute iliofemoral deep vein thrombosis (the CaVenT study): a randomised controlled trial. Lancet 2012; 379: 31-38. doi:10.1016/S01406736(11)61753-4. Epub 2011 Dec 13. PMID: 22172244 
[15] Herrera S, Comerota AJ, Thakur S et al. Managing iliofemoral deep venous thrombosis of pregnancy with a strategy of thrombus removal is safe and avoids post-thrombotic morbidity. J Vasc Surg 2014; 59 (2): 456-464. doi:10.1016/j.jvs.2013.07.108. Epub 2013 Aug 16. PMID: 23958068
[16] Ho VT, Dua A, Lavingia K et al. Thrombolysis for Venous Thromboembolism During Pregnancy: A Literature Review. Vasc Endovascular Surg 2018; 52 (7): 527-534. doi:10.1177/1538574418777822. Epub 2018 May 27. PMID: 29804522 